\title{
Obituary
}

\section{Professor Fergus Lowe}

On 10 December 2014, Professor Fergus Lowe died suddenly but peacefully in his Macclesfield home at the age of 68 years. Fergus Lowe was a leading figure in developing interventions and programmes to increase consumption of fruit and vegetables among young people and in developing protocols for testing their effectiveness. His main research interests included children's learning and language development and his scientific work in this domain has been published extensively in leading international journals.

Together with his colleague, Professor Pauline Horne, he developed a range of Food Dudes Behavioural Change programmes that were aimed at improving children's health, well-being and diet. These programmes were based on key principles from behavioural psychology such as of role modelling, rewards and repeated tasting to help children to enjoy eating fruit and vegetables and change their diets sustainably. The programme was trialled internationally and in 2012 it was established as a spin-out social enterprise company called Food Dudes Health.

Fergus Lowe served till his death as the Chair of the Board and Chief Executive of Food Dudes Health. He was a professor at the School of Psychology at Bangor University. He had previously served for more than 20 years as head of the psychology department, which grew under his leadership from a small school to one of the top research departments in the UK. He subsequently served as a Deputy Vice-Chancellor and as Acting ViceChancellor of Bangor University.

Fergus Lowe's research focused on behaviour change within the health domain and he served as an adviser to the British and Irish Governments as well as to the DirectorateGeneral for Agriculture and Rural Development in the European Commission on how to bring about change in children's eating habits. He served as the Vice-Chair of the EU's Scientific Expert Panel for the School Fruit Scheme and was a very active and committed speaker at many
European and international meetings. The latest advances in the Food Dudes programmes had been presented at the European Childhood Obesity Group meeting in Salzburg in November, shortly before Fergus Lowe's untimely death.

The Group of Experts (GREX) had been advising the European Commission on the evaluation and implementation of the School Fruit Scheme or - as it is referred to in its latest version - the School Fruit and Vegetables Scheme. Using his extensive experience from Food Dudes, Fergus was very active in the development of recommendations of the so-called accompanying measures. The Commission used this somewhat artificial but important term to denote all of the learning and teaching activities that are key elements of successful school fruit schemes, if they are to result in lasting behavioural change in the form of increased uptake of fruit and vegetables. The long hours spent in Brussels were, for all the members, also social moments and a time for meeting informally over a meal and a drink. Fergus will be remembered as a true gentleman who was open to socializing in breaks and after meetings. Fergus was excellent in his professional life but also a person of many interests and a very good conversation partner. The GREX members will certainly miss the professional achievement as well as the social moments in Brussels. We know that his work has contributed significantly to the advancement of the science of behavioural dietary change and to the ongoing improvement of the EU school fruit scheme.

Bent Egberg Mikkelsen Associate Editor and Past President of the GREX Email: bemi@plan.aau.dk

On behalf of the GREX (Bela Franchini, Saida Barnat, Margherita Caroli, Loes Neven, Mario Mazzocchi, Athena Linos, Victoria Anna Kovacs and Martine Padilla) and the GREX Secretariat (Giuliana Keller) 